\title{
Combining Static Analysis and Model Checking for Systems Employing Commutative Functions ${ }^{\star}$
}

\author{
A. Prasad Sistla and Min Zhou \\ University of Illinois at Chicago \\ \{sistla, mzhou\}@cs.uic.edu
}

\begin{abstract}
The two main hindrances for a wider application of the model checking approach for verification of concurrent and distributed systems are the state explosion problem and its limitation in handling infinite state systems. We consider a class of infinite state systems given by certain types of Transition Diagrams (TDs), called simple TDs, that employ commutative functions for updating variables. For such systems, we presented model checking methods that combine bi-simulation reductions with static analysis. The new methods are extensions of earlier methods where static analysis was not employed. These methods can be applied to a wider class of systems.
\end{abstract}

\section{Introduction}

The two main hindrances for a wider application of the model checking approach for verification concurrent and distributed programs are the state explosion problem and its limitation in handling infinite state systems. In our earlier work [7, we considered a class of infinite state systems given by certain types of Transition Diagrams (TDs), called simple TDs, that employ commutative functions for updating variables. For these systems, we presented model checking methods that use reductions with respect to a class of bi-simulation relations which can be checked on-the-fly. Experimental results showing the effectiveness of these approaches were also given. In this paper, we further extend these methods by combining them with static analysis to define larger bi-simulation relations. We give some applications of these results.

We assume that the concurrent program is given by a Transition Diagram (TD) 4 which is an edge labeled directed graph. Each edge label consists of a condition, called guard, and an action which is a concurrent assignment of values to variables. A TD is called a simple TD if the expression that is assigned to a variable $x$ is either a constant, or a variable, or of the form $f(x)$ where $f$ is a unary function. Further more, we require that the functions, that are used, are mutually commutative, that is, for any two functions $f, g, f g=g f$. We consider only simple TDs. It should be noted that such TDs are as powerful as Turing machines.

* This work is supported in part by the NSF grants CCR-9988884 and CCR-0205365.

F. Wang (Ed.): FORTE 2005, LNCS 3731, pp. 68-82 2005.

(C) IFIP International Federation for Information Processing 2005 
In [7, we defined an infinite sequence of non-decreasing bi-simulations, for simple TDs. These bi-simulations, denoted as $\sim_{k}$ for each $k \geq 0$, are defined over the symbolic state graph of the TD. Some of the techniques of 7 were implemented and applied to some practical protocols such as the sliding window protocol, producer-consumer protocols, etc.

In this paper, we extend the methods of [7] by combining them with simple techniques from static analysis. The combination of these techniques allows us to model check a wider class of programs, i.e., TDs. In the definition of $\sim_{k}$ introduced in [7], we defined two symbolic states $s$ and $t$ to be equivalent with respect to $\sim_{k}$ if the predicates obtained by instantiating certain predicate templates in these two states are equivalent; these templates are derived from the guards of the transitions of the TD and also from the temporal formula that one wants to check. The equivalence of the predicates assumes an implicit universal quantification over their free variables. We show that by analyzing the TD in advance, the domain over which the universal quantifiers range can be reduced. We actually give methods for obtaining constraints that restrict the domains of the quantifiers. This allows more states to be considered equivalent.

We give some applications of the above results. First, we consider simple TDs over integer domains where the functions updating the variables increment or decrement them by some constants. For such TDs, we show that we can define bi-simulations directly on their standard reachability graphs. For many integer TDs, we show that our method can be used to obtain finite quotient structures. We believe that our results can be applied to analyze real examples involving processes communicating through queues at certain level of abstraction.

Finally, we also show how the commutative requirement for simple TDs can be relaxed.

The paper is organized as follows. Section 2 briefly reviews the approach given in [7] and defines the notation. Section 2 also proves additional properties of the bi-simulations that were defined in [7. Section 3 gives methods that combine static analysis and bi-simulations. Section 5 shows how the commutativity requirement can be relaxed. Section [6] disusses related work.

\section{Background and Notation}

\subsection{Transition Diagram}

A TD is a triple $G=(Q, X, E)$ such that $Q$ denotes a set of nodes, $X$ is a set of variables, and $E$ is a set of transitions which are quadruples of the form $\left\langle q, C, \Lambda, q^{\prime}\right\rangle$ where $q, q^{\prime} \in Q, C$ is a condition involving the variables in $X$ and $\Lambda$ is a set of assignments of the form $x:=\rho$ where $x \in X$ and $\rho$ is an expression involving the variables in $X$. For a transition $\left\langle q, C, \Lambda, q^{\prime}\right\rangle$, we call $C$ the condition part or guard of the transition and $\Lambda$ the action part of the transition and we require that $\Lambda$ contains at most one assignment for each variable.

For any node $q$ of $G$, we let guards $(q)$ denote the set of guards of transitions from the node $q$. We also let guards $(G)$ denote the set of guards of all transitions of $G$. 
The semantics of a TD is defined in the usual way. A state of a TD $G=$ $(Q, X, E)$ is a pair $(q, h)$ where $q \in Q$, called location, and $h$ is an evaluation of $X$. A transition $e=\left(q_{1}, C, \Lambda, q_{2}\right)$ is enabled in the state $(q, h)$ if $q=q_{1}$ and the condition $C$ is satisfied by $h$. Let $s=(q, h)$ be a state and $e=\left(q_{1}, C, \Lambda, q_{2}\right)$ be a transition that is enabled in $s$. The transition $e$ can be executed in the state $s$ to obtain a successor state which is defined in the usual way.

A path in $G$ from node $q$ to node $r$ is a sequence of transitions starting with a transition from $q$ and ending with a transition leading to $r$ such that each successive transition starts from the node where the preceding transition ends. The left part of figure 1 shows a TD with 3 nodes. Notice that the transitions $t_{1}$ and $t_{2}$ both have empty guards meaning that they are always enabled.

\subsection{Kripke Structures, Bi-simulation, etc.}

A labeled Kripke structure $H$ over a set of atomic propositions $A P$ and over a set of labels $\Sigma$ is a triple $(S, R, L)$ where $S$ is a set of states, $R \subseteq S \times \Sigma \times S$ and $L: S \rightarrow 2^{A P}$ associates each state with a set of atomic propositions. The Kripke structure $H$ is said to be deterministic if for every $s \in S$ and every $\alpha \in \Sigma$ there exists at most one $s^{\prime} \in S$ such that $\left(s, \alpha, s^{\prime}\right) \in R$.

For the Kripke structure $H=(S, R, L)$, an execution/computation $\sigma$ is an infinite sequence $s_{0}, e_{0}, s_{1}, e_{1}, \ldots, s_{i}, e_{i}, \ldots$ of alternating states and labels in $\Sigma$ such that for each $i \geq 0,\left(s_{i}, e_{i}, s_{i+1}\right) \in R$. A finite execution/computation is a finite sequence of the above type ending in a label in a state. Corresponding to a finite execution $\sigma=s_{0}, e_{0}, s_{1}, \ldots, s_{m}$ let $\operatorname{trace}(\sigma)$ denote the finite sequence $L\left(s_{0}\right), e_{0}, \ldots, L\left(s_{i}\right), e_{i}, \ldots L\left(s_{m}\right)$. The length of a finite trace is the number of transitions in it. For any integer $k>0$, let Finite_Traces ${ }_{k}(H, s)$ denote the set of finite traces of length $k$ from $s$.

Let $H=(S, R, L)$ and $H^{\prime}=\left(S^{\prime}, R^{\prime}, L^{\prime}\right)$ be two structures over the same set of atomic propositions $A P$ and the same set $\Sigma$ of labels. A relation $B \subseteq S \times S^{\prime}$ is a bi-simulation between $H$ and $H^{\prime}$ iff for all $s \in S$ and $s^{\prime} \in S^{\prime}$, if $\left(s, s^{\prime}\right) \in B$, then $L(s)=L^{\prime}\left(s^{\prime}\right)$ and the following conditions hold: (a) for every $\left(s, \alpha, s_{1}\right) \in R$, there exists a state $s_{1}^{\prime} \in S^{\prime}$ such that $\left(s^{\prime}, \alpha, s_{1}^{\prime}\right) \in R^{\prime}$ and $\left(s_{1}, s_{1}^{\prime}\right) \in B$; (b) similarly, for every $\left(s^{\prime}, \alpha, s_{1}^{\prime}\right) \in R^{\prime}$, there exists a state $s_{1} \in S$ such that $\left(s, \alpha, s_{1}\right) \in R$ and $\left(s_{1}, s_{1}^{\prime}\right) \in B$. A sub-bi-simulation between $H$ and $H^{\prime}$ is any subset of a bisimulation relation between $H$ and $H^{\prime}$.

Suppose $G=(Q, X, E)$ is a TD and $u$ is a state. The semantics of $G$ starting from the state $u$ can be defined by a Kripke structure $\operatorname{Reach}(G, u)=(S, R, L)$ over the set of atomic propositions $A P$ and the set of labels $E$ as follows: $S$ is the set of reachable states obtained by executing the TD $G$ from $u ; R$ is the set of triples $\left(s, e, s^{\prime}\right)$ such that the transition $e \in E$ is enabled in state $s$ and $s^{\prime}$ is obtained by executing $e$ in state $s$; for any $s \in S, L(s)$ is the set of atomic propositions in $A P$ that are satisfied in $s$. Computations of the TD $G$ are computations of $\operatorname{Reach}(G, u)$. Let $\pi=e_{0}, e_{1}, \ldots, e_{m-1}$ be a path in $G$ from node $q$ and let $s_{0}=\left(q, h_{0}\right)$ be a state. We say that $\pi$ is feasible from $s_{0}$ if there exists a finite computation of the form $s_{0}, e_{0}, s_{1}, \ldots, e_{m-1}$ in the $\operatorname{Reach}\left(G, s_{0}\right)$. In this case, we say that $s_{m}$ is the state obtained by executing the path $\pi$ from 
$s_{0}$. For the TD given in 1, it is easy to see that the reachability graph from the state $(0, h)$, where $h$ assigns 0 to all the variables, is infinite since $x, y$ can grow arbitrarily large.

Let $B$ be any sub-bi-simulation relation from $\operatorname{Reach}(G, u)$ to itself. Instead of constructing $\operatorname{Reach}(G, u)$, we can construct a smaller structure using the relation $B$. We incrementally construct the structure by executing $G$ starting from $u$. Whenever we get a state $w$ by executing a transition from an already reached state $v$, we check if there exists an already reached state $w^{\prime}$ such that $\left(w, w^{\prime}\right)$ or $\left(w^{\prime}, w\right)$ is in $B$; if so, we simply add an edge to $w^{\prime}$ otherwise we include $w$ into the set of reached states and add an edge to $w$. This procedure is carried until no more new nodes can be added to the set of reached states. We call the resulting structure the reduction of $\operatorname{Reach}(G, u)$ with respect to $B$. This reduction has the property that no two states in it are related by $B$. The number of states in this reduction may not be unique and may depend on the order of execution of the enabled transitions. However, if $B$ is an equivalence relation then the number of states in the reduction is unique and equals the number of equivalence classes of $S$ with respect to $B$.

We use the temporal logic CTL* [1] to specify properties of $\operatorname{Reach}(G, u)$. Each atomic proposition in the formulas is a predicate involving variables in $X$ or the special variable $l c$ which refers to the nodes of $G$. We let $A P$ be the set of predicates that appear in the temporal formula that we want to check. The atomic propositions refering to $l c$ are of the form $l c=q$ where $q$ is a node of $G$. For any formula or predicate $p$, we let $\operatorname{var}(p)$ denote the set of variables appearing in it.

If $K$ is a reduction of $\operatorname{Reach}(G, u)$ with respect to a sub-bisimulation relation then a state which is present in both $\operatorname{Reach}(G, u)$ and $K$ satisfies the same set of $\mathrm{CTL}^{*}$ formulas in both structures. Also, any two states in $\operatorname{Reach}(G, u)$, that are related by a sub-bi-simulation, satisfy the same set of $\mathrm{CTL}^{*}$ formulas.

\subsection{Symbolic Graph}

For an expression $\Phi$, we use $\Phi\{\beta / \alpha\}$ denote the expression obtained from $\Phi$ by substituting $\beta$ for $\alpha$.

Let $G=(Q, X, E)$ be a TD, $u=\left(q_{0}, h_{0}\right)$ be the initial state of $G$. $\operatorname{Sym} \_\operatorname{Reach}(G, u)=\left(S^{\prime}, R^{\prime}, L^{\prime}\right)$, called symbolic graph, is a Kripke structure obtained by the symbolic execution of $G$ starting from $u$. Each state $s$ in $S^{\prime}$, called a symbolic state, is a triple of the form (s.lc, s.val, s.exp) where s.lc $\in Q$, s.val is an evaluation of the variables in $X$ and s.exp is a function that assigns each variable $x$ an expression which involves only the variable $x$ itself. Intuitively, s.lc denotes the node in $Q$ where the control is, $\operatorname{s.val}(x)$ denotes the latest constant assigned to $x$ and $\operatorname{sexp}(x)$ denotes the composition of functions that were applied to $x$ since then. It is easy to see that a symbolic state $s$ corresponding to the actual state $(s . l c, h)$ where $h(x)=s . \exp (x)\{\operatorname{s.val}(x) / x\}$ for each $x \in X$. We let act_state $(s)$ denote the actual state corresponding to the symbolic state $s$. Each member of $R^{\prime}$ is a triple of the form $\left(s, e, s^{\prime}\right)$ where $s \in S$ and $e \in E$ that is enabled in $s$ and $s^{\prime} \in S$ is the successor of $s$ after the execution of $e . L^{\prime}$ is a 
labeling function that associates with each symbolic state $s$, the set of predicates in $A P \cup \operatorname{guards}(G)$ that are satisfied in the corresponding actual state. Note that the predicate $l c=q$ is satisfied in a symbolic state $s$, if $s . l c=q$. We say that a transition $e$ is enabled in a symbolic state $s$ if it is enabled in the corresponding actual state, i.e., in the state act_state $(s)$.

The successor states of a symbolic state $s$ are defined as follows. Assume that a transition $e=\left(q, C, \Lambda, q^{\prime}\right)$ is enabled in $s$. The new symbolic state $s^{\prime}$ obtained by executing $e$ from $s$ is defined as follows: $s^{\prime} . l c=q^{\prime}$ and for each variable $x$, if there is no assignment to $x$ in $\Lambda$ then $s^{\prime} \cdot \operatorname{val}(x)=\operatorname{s.val}(x)$ and $s^{\prime} \cdot \exp (x)=s \cdot \exp (x)$. If there is an assignment of the form $x:=c$ where $c$ is a constant then $s^{\prime} \cdot \operatorname{val}(x)=c$ and $s^{\prime} \cdot \exp (x)=x$. If there is an assignment of the form $x:=\psi(x)$ in $\Lambda$ then $s^{\prime} \cdot \operatorname{val}(x)=\operatorname{s\cdot val}(x)$ and $s^{\prime} \cdot \exp (x)=\psi(\operatorname{sexp}(x))$; that is the value remains unchanged and the new expression is obtained by applying the function $\psi$ to the old expression. If there is an assignment of the form $x:=y$ in $\Lambda$ then $s^{\prime} \cdot \operatorname{val}(x)=\operatorname{s.val}(y)$ and $s^{\prime} \cdot \exp (x)=\operatorname{sexp}(y)\{x / y\}$; that is the value of $\operatorname{s.val}(y)$ is copied and the expression of $y$ in $s$ is also copied after replacing every $y$ by $x$ in the expression. If $s^{\prime}$ is obtained by executing an enabled transition $e$ from a state $s$ in $S^{\prime}$, then $s^{\prime}$ is a state in $S^{\prime}$ and $\left(s, e, s^{\prime}\right) \in R^{\prime}$.
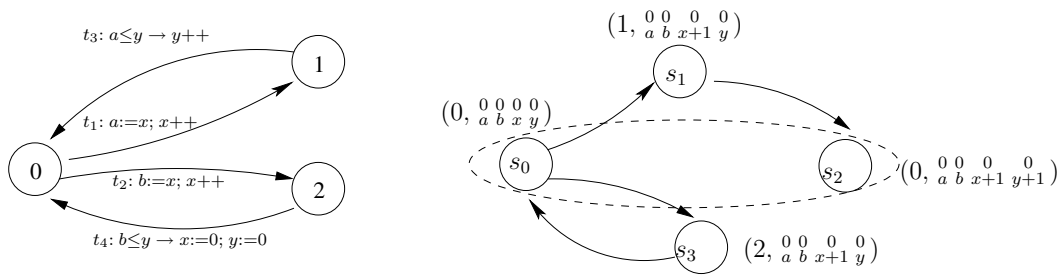

Fig. 1. Example of a TD and its reduced symbolic state graph with respect to $\sim_{0}$

For the TD given in the left part of figure 1 with initial value $a=b=x=$ $y=0$, the right part of figure 1 gives part of its symbolic state graph. The initial state $s_{0}$ is $\left(0, \begin{array}{llll}0 & 0 & 0 & 0 \\ a & b & x & y\end{array}\right)$, where the first 0 denotes the node, the vectors $(0,0,0,0)$ and $(a, b, x, y)$ represent the functions $s_{0} . v a l$ and $s_{0} . \exp$ respectively.

It should be noted that an actual state may be represented by several symbolic states which are obtained by different execution paths in our symbolic state graph.

\subsection{Predicate Templates and Definition of $\sim_{0}$}

Let $\pi$ be a path in $G$. Such path denotes a possible execution in $G$. For the path $\pi$, we define a function depends $s_{\pi}$ from $X$ to $X \cup\{*\}$ as follows. Intuitively, if $\operatorname{depend}_{\pi}(x)$ is a variable, say $y$, this means the value of $x$ after the execution of $\pi$ depends on the value of $y$ before the execution of $\pi$. We define depends $s_{\pi}$ inductively on the length of $\pi$. If $\pi$ is a single transition $\left\langle q, C, \Lambda, q^{\prime}\right\rangle$ then depend $s_{\pi}(x)$ is given as follows: if $\Lambda$ has the assignment 
$x:=y$ then $\operatorname{depends}_{\pi}(x)=y$; if $\Lambda$ has no assignment to $x$ or has an assignment of the form $x:=\psi(x)$ then $\operatorname{depend}_{\pi}(x)=x$; when $x$ is assigned a constant, depend $s_{\pi}(x)=*$. If $\pi$ is the path consisting of $\pi_{1}$ followed by $\pi_{2}$ then depend $s_{\pi}$ is defined as follows: for each $x \in X$, if depend $_{\pi_{\pi_{2}}}(x)$ is a variable then depend $s_{\pi}(x)=$ depend $s_{\pi_{1}}\left(\right.$ depend $\left._{\pi_{2}}(x)\right)$, otherwise depend $s_{\pi}(x)=*$.

A predicate template is a pair $(p, f)$ where $p$ is a predicate and $f$ is function from $X$ to $X \cup\{*\}$. With each node $q$ we associate a set ptemplates $(q)$ defined as follows: ptemplates $(q)=\left\{\left(\right.\right.$ p, depend $\left.s_{\pi}\right): \pi$ is a path from node $q$ to some node $r$ and $p \in$ guards $(r) \cup A P\}$. An efficient method to compute ptemplates $(q)$ without examining all the paths from $q$ is given in [7].

Now we define the instantiation of a predicate template in a symbolic state. Suppose $s$ is a state of the symbolic state graph $\operatorname{Sym} \_\operatorname{Reach}(G, u),(p, f)$ is a predicate template and $x_{1}, x_{2}, \cdots, x_{n}$ are variables appearing in $p$. Let $p^{\prime}$ be the predicate obtained by replacing every occurrence of the variable $x_{i}$ (for $1 \leq i \leq n)$, for those $x_{i}$ such that $f\left(x_{i}\right) \neq *$, by the expression $\operatorname{sexp}\left(y_{i}\right)\left\{x_{i} / y_{i}\right\}$ where $y_{i}$ is the variable $f\left(x_{i}\right)$. Note that the variables $x_{i}$ for which $f\left(x_{i}\right)=*$ are not replaced. We define $(p, f)[s]$ to be $p^{\prime}$ as given above.

Definition 1. Define relation $\sim_{0}$ as follows: For any two states $s$ and $t, s \sim_{0} t$ iff $s . l c=t . l c$, s.val $=$ t.val and for each $(p, f) \in \operatorname{ptemplates}(s . l c),(p, f)[s] \equiv$ $(p, f)[t]$ is a valid formula.

The following theorem is proved in [7] and its proof crucially depends on the commutativity requirement.

Theorem 1. $\sim_{0}$ is a bi-simulation on the symbolic state graph $\operatorname{Sym} \_$Reach $(G, u)$.

\subsection{Extended Predicate Templates and $\sim_{k}$}

In order for two symbolic states $s$ and $t$ to be related by $\sim_{0}$, we required that $s . l c=t . l c$ and for every guard $p$ of a transition from a node $r$ that is reachable from s.lc in the TD and for every template $(p, f) \in$ ptemplates $(s . l c)$, we required that the instantiations of $(p, f)$ in $s$ and $t$ be equivalent. This requirement can be relaxed as follows. If none of the paths leading to $r$ is feasible then, in the above requirement, we do not need to consider templates of the form $(p, f)$ where $p$ is a guard of some transition from $r^{\prime}$. Now we define a bi-simulation relation $\sim_{k}$ in which we relax the equivalence condition. Roughly speaking, in the definition of $\sim_{k}$, we require $s$ and $t$ to be equivalent with respect to guards of node $r$ only when there exists a path of length at least $k$ leading to $r$ which is feasible for both $s$ and $t$.

Let $\pi$ be a path in $G,|\pi|$ be the length of $\pi$ and $p$ be any predicate. Also, let $\pi^{\prime} \circ \pi^{\prime \prime}$ denote the path $\pi^{\prime}$ followed by $\pi^{\prime \prime}$. Now we define the weakest precondition of $p$ with respect to $\pi$, denoted by $W P(\pi, p)$ as follows.

$$
W P(\pi, p)= \begin{cases}p & \text { if }|\pi|=0 \\ p\left\{\rho_{i} / x_{i}\right\} & \text { if }|\pi|=1 \wedge \text { the assignments are } x_{i}:=\rho_{i} \\ W P\left(\pi^{\prime}, W P(e, p)\right) & \text { if } \pi=\pi^{\prime} \circ e\end{cases}
$$


It has been shown 7 that if path $\pi$ of $G$ is feasible from state $s$, and $s^{\prime}$ is the state obtained by executing $\pi$ from $s$, then $s^{\prime}$ satisfies $p$ iff $s$ satisfies $W P(\pi, p)$.

Suppose $\pi=e_{0} \circ e_{1} \circ \ldots, e_{k-1}$ be a path in $G$ and for $0 \leq i<k, C_{i}$ is the guard of the transition $e_{i}$. For each $i, 0<i \leq k$, let $\pi(i)$ denote the prefix of $\pi$ consisting of the first $i$ transitions, i.e., the path from $e_{0}$ to $e_{i-1}$. Define $\operatorname{Cond}(\pi)$ to be the predicate $C_{0} \wedge \bigwedge_{0<i<k} W P\left(\pi(i), C_{i}\right)$. It has be shown [7] that a path $\pi$ of $G$ is feasible from state $s$ iff $s$ satisfies the predicate $\operatorname{Cond}(\pi)$.

The set extended_templates $(q, k)$ is defined to be the set of triples which are of the form $\left(\operatorname{Cond}\left(\pi^{\prime \prime}\right), W P\left(\pi^{\prime \prime}, p\right)\right.$, depend $\left.s_{\pi^{\prime}}\right)$ where $\pi^{\prime}, \pi^{\prime \prime}$ are paths such that $\pi^{\prime} \pi^{\prime \prime}$ (i.e., $\pi^{\prime}$ followed by $\pi^{\prime \prime}$ ) is a path from $q$ to some node $r$, the length of $\pi^{\prime \prime}$ is $k$ and $p \in \operatorname{guards}(r) \cup A P$. Similar to ptemplates $(q)$, we have an efficient method to compute extended_templates $(q, k)$ without examining all the paths from $q$. Note that unlike ptemplates $(q)$, extended_templates $(q, k)$ are triples.

Definition 2. Define relation $\sim_{k}$ on the states of $\operatorname{Sym}{ }_{-} \operatorname{Reach}(G, u)$ as follows. $\sim_{k}$ is the set of all pairs $(s, t)$ where $s, t$ are states of $\operatorname{Sym}$ Reach $(G, u)$ such that:

1. $s . l c=t . l c$ and s.val $=$ t.val

2. Finite_Traces ${ }_{k}(s)=$ Finite_Traces $_{k}(t)$ and

3. for every $\left(p_{1}, p_{2}, f\right) \in$ extended_templates $(s . l c, k)$ the formula $\left(\left(p_{1}, f\right)[s] \wedge\right.$ $\left.\left(p_{1}, f\right)[t]\right) \supset\left(\left(p_{2}, f\right)[s] \equiv\left(p_{2}, f\right)[t]\right)$ is a valid formula.

Theorem 2, 3] and 4 are from [7].

Theorem 2. For each $k>0, \sim_{k}$ is a bi-simulation relation.

Theorem 3. For every $k \geq 0$, every $T D G$, and for every set $A P$ of atomic predicates, $\sim_{k} \subseteq \sim_{k+1}$.

Theorem 4. For every $k \geq 0$, there exists a TD $G$ and a set of atomic predicates AP for which the above containment is strict, i.e. $\sim_{k} \subset \sim_{k+1}$.

\subsection{Relationships Among the Bisimulation Relations}

In this sub-section we state theorems strengthing previously stated results. We can strengthen theorem 4 as follows.

Theorem 5. Given any integer $k \geq 0$, there exists a TD such that $\sim_{k+1}$ gives finite quotient structure while $\sim_{k}$ does not.

Theorem 6. There exists a TD for which $\forall k>0, \sim_{k} \subset \sim_{k+1}$.

\section{Using Static Analysis to Constrain the Quantifiers' Range}

Recall that in the definition of $\sim_{0}$, for $s \sim_{0} t$, we require that for each template $(p, f) \in$ ptemplates $(s . l c),(p, f)[s] \equiv(p, f)[t]$. It should be noted that there is an implicit universal quantification on variables when we assert the validity of the 


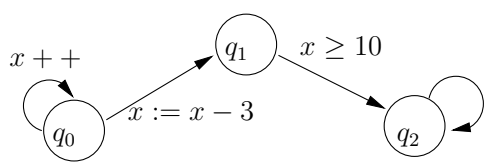

Fig. 2. Example of a TD which can get finite reduction by a simple static analysis

equivalence $(p, f)[s] \equiv(p, f)[t]$. This universal quantification can be restricted to range over a smaller set of values in some cases. By this change, we get another bi-simulation relation which is larger than $\sim_{0}$.

Example: Consider figure 2 which is a TD over a single integer variable $x$. The transition from $q_{1}$ to $q_{2}$ has null action and the guards of all other transitions are "true". The initial state is $u=\left(q_{0}, h\right)$ where $h(x)=0$. For this TD, it can be shown that $\sim_{0}$ is the identity relation and hence the reduction of $\operatorname{Sym} \_$Reach $\left(G_{1}, u\right)$ with respect to $\sim_{0}$ is infinite. By a simple static analysis, one can show that the value of $x$, when control is at node $q_{1}$, is greater than or equal to -3 . We show below that, the quantifier over $x$ correspoding to the predicate template for the predicate $x \geq 0$ can be restricted to the values of $x \geq-3$. Using this modification, we define another bi-simulation $\sim_{0}^{\prime}$ which gives a finite reduction for $\operatorname{Sym} \_$Reach $\left(G_{1}, u\right)$; the number of states in this reduction is 13 .

Now consider simple TD $G$ and any state $s$ in $\operatorname{Sym} \_\operatorname{Reach}(G, u)$. Let $(p, f)$ be any predicate template in ptemplates $($ s.lc) such that $p \in \operatorname{guards}(G) \cup A P$. Let $\left\{x_{1}, \ldots, x_{n}\right\}$ be all the variables in $\operatorname{var}(p)$ and $Q_{p} \subseteq Q$ be as defined below: if $p \in A P$ then $Q_{p}=Q$, otherwise $Q_{p}$ is the set of all nodes $q$ in $G$ such that $p \in \operatorname{guards}(q)$.

Let $G^{\prime}$ be the TD obtained from $G$ by replacing all guards in its transitions by the constant predicate True. Now let $T_{p, s}$ be the set $\{h: h$ is a valuation and for some $q \in Q_{p}$ the state $(q, h)$ is reachable from the state $(s . l c, s . v a l)$ in $\left.\operatorname{Reach}\left(G^{\prime}, u\right)\right\}$. Essentially, $T_{p, s}$ is the set of valuations for the variables in $X$ when the control is at some node in $Q_{p}$ when the $T D G^{\prime}$ is executed starting from the state $(s . l c, s . v a l)$. Let $g_{p, s}$ be a formula, with free variables from $X$, that defines the set $T_{p, s}$, i.e., $T_{p, s}$ is exactly the set of valuations that satisfy the constraint given by $g_{p, s}$. (Note that we are assuming that the assertion logic is expressive enough to express this set). Essentially, $g_{p, s}$ defines a constraint on the values of the variables when the control is at some node in $Q_{p}$. Let $\sim_{0}^{\prime}$ be the set of $(s, t)$ such that $s, t$ are symbolic states, s.lc $=t . l c$, s.val $=t . v a l$, and for all $(p, f) \in$ ptemplates $(s . l c)$ the formula $g_{p, s} \supset((p, f)[s] \equiv(p, f)[t])$ is valid. Thus we see that the quantifiers over the variables in $X$ are restricted to the valuations that satisfy $g_{p, s}$, i.e., to the valuation in $T_{p, s}$. Observe that $T_{p, s}=T_{p, t}$; thus it does not matter whether we use $s$ or $t$ for obtaining these sets and the formulas $g_{p, s}$. Note that if we replace $g_{p, s}$ by True then we get $\sim_{0}$. From this, it should be easy to see that $\sim_{0}^{\prime} \supseteq \sim_{0}$.

Theorem 7. $\sim_{0}^{\prime}$ is a bi-simulation on symbolic state graph Sym_Reach $(G, u)$.

Proof: Assume that $s \sim_{0}^{\prime} t$. Clearly, s.lc $=t . l c$ and s.val $=t . v a l$. Let $p \in(A P \cup$ $\operatorname{guards}(s . l c))$. From the definition of ptemplates(s.lc), we see that $\left(p, f_{i d}\right) \in$ 
ptemplates $(s . l c)$. By the definition of $\sim_{0}^{\prime}, g_{p, s} \supset\left(p, f_{i d}\right)[s] \equiv\left(p, f_{i d}\right)[t]$ is valid. The tuple of values of variables defined by s.val belongs to $T_{p, s}$ and hence it satisfies the formula $g_{p, s}$. As a consequence, the two predicates $\left(p, f_{i d}\right)[s]$ and $\left(p, f_{i d}\right)[t]$ evaluate to the same truth value, when the variables in them are substituted by the values given by $\operatorname{s.val}(x)$. It is also not difficult to see that these truth values, respectively, are the truth value of the predicate $p$ in the actual states act_state $(s)$ and act_state $(t)$. It is easy to see that the symbolic state $s$ satisfies $p$ iff $t$ satisfies $p$. Since this property holds for every $p \in(A P \cup \operatorname{guards}(s . l c))$, it is easily seen that $L^{\prime}(s)=L^{\prime}(t)$ and every transition $e$ from node $s . l c$ is enabled in $s$ iff it is enabled in $t$. Now assume $\left(s, e, s^{\prime}\right) \in R^{\prime}$ which means there is an enabled transition $e$ in $s$ and $s^{\prime}$ is reached after the execution of $e$. From above analysis, we know that $e$ is also enabled in $t$. Let $t^{\prime}$ be the symbolic state obtained by executing $e$ from $t$. We show that $s^{\prime} \sim_{0}^{\prime} t^{\prime}$. Obviously, $s^{\prime} . l c=t^{\prime} . l c$ and $s^{\prime} . v a l=t^{\prime} . v a l$.

Now, we show that for all $\left(p, f^{\prime}\right) \in$ ptemplates $\left(s^{\prime} . l c\right), g_{p, s^{\prime}} \supset\left(\left(p, f^{\prime}\right)\left[s^{\prime}\right] \equiv\right.$ $\left.\left(p, f^{\prime}\right)\left[t^{\prime}\right]\right)$ is a valid formula. Consider a template $\left(p, f^{\prime}\right) \in \operatorname{ptemplates}\left(s^{\prime} . l c\right)$. From the definition of ptemplates $(s . l c)$, it is seen that there exists a $(p, f) \in$ ptemplates $(s . l c)$ satisfying the following conditions for every $x$ in $\operatorname{var}(p)$. These conditions are divided into five cases. The first case is when $f^{\prime}(x)=*$; in this case, $f(x)=*$ and the variable $x$ in $p$ remains unchanged when we obtain $(p, f)[s]$ and $\left(p, f^{\prime}\right)\left[s^{\prime}\right]$ from $p$. In the other cases $f^{\prime}(x) \neq *$, i.e., $f^{\prime}(x)$ is a variable. Let $f^{\prime}(x)$ be the variable $y$. The remaining cases depend on whether there is an assignment to $y$ in the action part of $e$ and if so, what is assigned to it. The second case is when there is no assignment for the variable $y$. In this case, $f(x)=y$ and $s^{\prime} \cdot \exp (y)=s \cdot \exp (y)$; hence the expressions substituted for $x$ in $p$ to obtain $(p, f)[s]$ and $\left(p, f^{\prime}\right)\left[s^{\prime}\right]$ are identical. The third case is when $y$ is assigned a constant; in this case, $f(x)=*$ and from the construction of Sym_Reach $(G, u)$, we see that $s^{\prime} \cdot \exp (y)=y$ and hence the expressions substituted for $x$ in $p$ to obtain $(p, f)[s]$ and $\left(p, f^{\prime}\right)\left[s^{\prime}\right]$ are both $x$ itself. The fourth case is when $y$ is assigned a variable $z$; in this case, $f(x)=z$ and $s^{\prime} \cdot \exp (y)=\operatorname{sexp}(z)\{y / z\}$; from this it should be easy to see that the expressions substituted for $x$ in $p$ to obtain $(p, f)[s]$ and $\left(p, f^{\prime}\right)\left[s^{\prime}\right]$ are identical.

In all above four cases, we see that the same expression is substituted for $x$ in $p$ to obtain both $(p, f)[s]$ and $\left(p, f^{\prime}\right)\left[s^{\prime}\right]$. The fifth and last case is when there is an assignment of the form $y:=\psi(y)$ where $\psi$ is a unary function; in this case, $f(x)=y$ and $s^{\prime} \cdot \exp (y)=\psi(\operatorname{sexp}(y))$; since the function $\psi$ is commutative with the functions appearing in $\operatorname{sexp}(y)$, it is easy to see that $s^{\prime} \cdot \exp (y)=s \cdot \exp (y)\{\psi(y) / y\}$; it should be noted that the variable $x$ in $p$ is substituted by $\operatorname{sexp}(y)\{x / y\}$ and by $s^{\prime} \cdot \exp (y)\{x / y\}$ respectively to obtain $(p, f)[s]$ and $\left(p, f^{\prime}\right)\left[s^{\prime}\right]$. From the above observations, we see that the expression substituted for $x$ in $p$ to obtain $\left(p, f^{\prime}\right)\left[s^{\prime}\right]$ is $\operatorname{s.exp}(y)\{\psi(x) / y\}$. Let $x_{1}, \ldots, x_{n}$ be the variables in $\operatorname{var}(p)$ to which this last case applies. For each $i=1, \ldots, n$, let $f^{\prime}\left(x_{i}\right)=y_{i}$ and $y_{i}:=\psi_{i}\left(y_{i}\right)$ be the assignment to $y_{i}$ in the action part of $e$.

From the above observations, we see that the expression substituted for $x_{i}$ in $p$ to obtain $(p, f)[s]$ is $\operatorname{s.exp}\left(y_{i}\right)\left\{x_{i} / y_{i}\right\}$, while the expression substituted for $x$ in 
$p$ to obtain $\left(p, f^{\prime}\right)\left[s^{\prime}\right]$ is $s . \exp \left(y_{i}\right)\left\{\psi_{i}\left(x_{i}\right) / y_{i}\right\}$. Using this fact for each $i=1, \ldots, n$ and the fact that in all the first four cases the same expressions are substituted for $x$ in $p$ to obtain both $(p, f)[s]$ and $\left(p, f^{\prime}\right)\left[s^{\prime}\right]$, it is not difficult to see that $\left(p, f^{\prime}\right)\left[s^{\prime}\right]=(p, f)[s]\left\{\psi_{1}\left(x_{1}\right) / x_{1}, \ldots, \psi_{n}\left(x_{n}\right) / x_{n}\right\}$. By the same argument, we see that $\left(p, f^{\prime}\right)\left[t^{\prime}\right]=(p, f)[t]\left\{\psi_{1}\left(x_{1}\right) / x_{1}, \ldots, \psi_{n}\left(x_{n}\right) / x_{n}\right\}$.

Now we relate the sets $T_{p, s}, T_{p, s^{\prime}}$, and consequently, the formulas $g_{p, s}, g_{p, s^{\prime}}$. Recall that $T_{p, s}$ denotes the set of valuations for variables in $X$ when control is in some location in $Q_{p}$ in a computation of the TD $G^{\prime}$ starting from the state (s.lc, s.val). Using the same argument as given above, it is easy to see that for every $h^{\prime} \in T_{p, s^{\prime}}$ there exists a $h \in T_{p, s}$ satisfying the following condition: for $1 \leq i \leq n, h\left(x_{i}\right)=\psi_{i}\left(h\left(x_{i}\right)\right)$; for every $z \in X-\left\{x_{i}: 1 \leq i \leq n\right\}, h(z)=h^{\prime}(z)$. From this it follows that the formula $g_{p, s^{\prime}} \supset g_{p, s}\left\{\psi_{1}\left(x_{1}\right) / x_{1}, \ldots, \psi_{n}\left(x_{n}\right) / x_{n}\right\}$ is a valid formula.

Since, $g_{p, s} \supset(p, f)[s] \equiv(p, f)[t]$ is valid, its validity holds even when we replace each $x_{i}$ by $\psi\left(x_{i}\right)$ for $i=1, \ldots, n$. When this replacement is done, we get that $g_{p, s}\left\{\psi_{1}\left(x_{1}\right) / x_{1}, \ldots, \psi_{n}\left(x_{n}\right) / x_{n}\right\} \supset\left(\left(p, f^{\prime}\right)\left[s^{\prime}\right] \equiv\left(p, f^{\prime}\right)\left[t^{\prime}\right]\right)$ is valid. Since $g_{p, s^{\prime}} \supset$ $g_{p, s}\left\{\psi_{1}\left(x_{1}\right) / x_{1}, \ldots, \psi_{n}\left(x_{n}\right) / x_{n}\right\}$ is a valid formula, we see that $g_{p, s^{\prime}} \supset\left(\left(p, f^{\prime}\right)\left[s^{\prime}\right] \equiv\right.$ $\left.\left(p, f^{\prime}\right)\left[t^{\prime}\right]\right)$ is a valid formula.

Now consider the the example TD $G_{1}$ given by figure 2 , Let $u=\left(q_{0}, h\right)$ where $h(x)=0$. Let $p$ be the guard $x \geq 10$ which is the guard of the transition from $q_{1}$ to $q_{2}$. Let $s$ and $t$ be two states in $\operatorname{Sym} \_$Reach $\left(G_{1}, u\right)$ with $s . l c=q_{0}, t . l c=q_{0}$, $s . \exp (x)=x+c$ and $t \cdot \exp (x)=x+d$. Observe that $c, d \geq 0$. It should be easy to see that $g_{p, s}$ is the constraint $x \geq-3$. The template $\left(x \geq 10, f_{i d}\right)$ is the only non-trivial predicate template in ptemplates $\left(q_{0}\right)$. From our definition of $\sim_{0}^{\prime}$, it is easily seen that $s \sim_{0}^{\prime} t$ iff the formula $x \geq-3 \supset(x+c \geq 10 \equiv$ $x+d \geq 10)$ is a valid formula. This gives us 13 equivalence classes and hence the reduction of $\operatorname{Sym} \_$Reach $(G, u)$ has 13 states. (Note that in the definition of $\sim_{0}$, the equivalence $(x+c \geq 10 \equiv x+d \geq 10)$ is required to hold for all values of $x$ including all negative values; due to this reason, $\sim_{0}$ is an identity relation as pointed out at the beginning of the section).

The above defintion of $\sim_{0}^{\prime}$ can be naturally extended to $\sim_{i}$ for every $i \geq 0$ giving us the relations $\sim_{i}^{\prime}$.

In the above definitions, we see that the formula $g_{p, s}$ depends on $p$, s.lc and s.val. Note that the set of possible values of s.val can be determined from static analysis of $G$. Essentially, for any variable $x, \operatorname{s.val}(x)$ is a constant that is assigned to $x$ in the action part of some transition. Since we ignore all the guards when we compute $T_{p, s}$, it is not difficult to see that $T_{p, s}$ or $g_{p, s}$ can be computed by static analysis of $G$. The number of values of s.val can be exponential. Many times we can get a constraining formula $g_{p, s}$ which is independent of s.val. For example, consider the example given in figure [3, a possible $g_{p, s}$ can be $\left(x_{1} \geq\right.$ $\left.0 \wedge x_{2} \geq 0\right)$ for every predicate $p$ and state $s$ since all the updating functions increment variables.

Now, we consider the TD $G$ given in figure 3 with initial state $\left(q_{0}, h\right)$ where $h\left(x_{1}\right)=h\left(x_{2}\right)=0$. We show that $\sim_{0} \subset \sim_{0}^{\prime} \subset \sim_{1}^{\prime}$ for this TD. First, it is easy to see that ptemplates $\left(q_{0}\right)=\left\{\left(x_{1}=0, f_{i d}\right),\left(x_{2}=0, f_{i d}\right),\left(x_{1} \geq 20, f_{i d}\right),\left(x_{2} \geq\right.\right.$ 


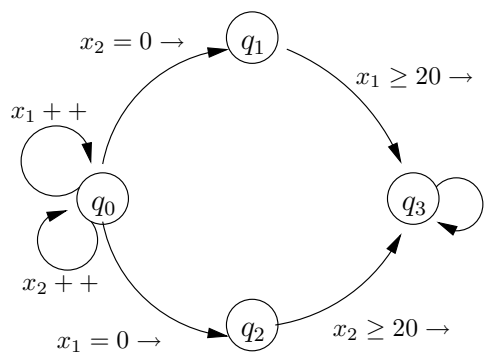

Fig. 3. Example of a TD for which $\sim_{0} \subset \sim_{0}^{\prime} \subset \sim_{1}^{\prime}$

$\left.\left.20, f_{i d}\right)\right\}$. For every state $s$ in $\operatorname{Sym} \_\operatorname{Reach}(G, u)$ and for every guard $p, g_{p, s}$ is the constraint $\left(x_{1} \geq 0 \wedge x_{2} \geq 0\right)$. Also, for any state $s$ in $\operatorname{Sym} \_\operatorname{Reach}(G, u)$ such that $s . l c=q_{0}$ it is the case that $\operatorname{s.val}\left(x_{1}\right)=\operatorname{s.val}\left(x_{2}\right)=0$. Let $s, t$ be any two states such that $s . l c=t . l c=0$. Let $s \cdot \exp \left(x_{1}\right)=x_{1}+c_{1}$ and $\operatorname{sexp}\left(x_{2}\right)=x_{2}+c_{2}$; similarly, let $t . \exp \left(x_{1}\right)=x_{1}+d_{1}$ and $\operatorname{s.exp}\left(x_{2}\right)=x_{2}+d_{2}$. Now, $s$ and $t$ are related by $\sim_{0}^{\prime}$ if the values $c_{1}, c_{2}, d_{1}, d_{2}$ are all $\geq 20$. (As in the previous example, the relation $\sim_{0}$ is simply the identity relation).

Now consider the relation $\sim_{1}^{\prime}$. From the definitions, it can be seen that extended_templates $\left(q_{0}, 1\right)=\left\{\left(x_{2}=0, x_{1} \geq 20, f_{i d}\right),\left(x_{1}=0, x_{2} \geq 20, f_{i d}\right),(\right.$ true, $\left.x_{1}=0, f_{i d}\right)$, (true, $\left.\left.x_{2}=0, f_{i d}\right)\right\}$. By a detailed combinatorial analysis, it can be shown that the set of states in $\operatorname{Sym} \_$Reach $(G, u)$ form into $21^{2}-21$ classes with respect to $\sim_{0}^{\prime}$ and 42 classes with respect to the bi-simulation $\sim_{1}^{\prime}$. In a more general case, if the constant in the transitions entering node $q_{3}$ is $c$ then the set of states of the form $\left(q_{0}, h\right)$ will be divided in to $(c+1)^{2}-(c+1)$ and $2(c+1)$ classes with respect to the bi-simulations $\sim_{0}^{\prime}, \sim_{1}^{\prime}$ respectively. Thus in the general case, the total number of states in the reductions with respect to $\sim_{0}^{\prime}, \sim_{1}^{\prime}$ are given by $(c+1)^{2}+2(c+1)$ and $3(c+1)+3$ respectively. Thus $\sim_{1}^{\prime}$ gives more reduction. In general, we can generalize the example by introducing $n$ arbitrary variables where $\sim_{1}^{\prime}$ has much more reduction than $\sim_{0}^{\prime}$.

The above analysis assumes that the set $T_{p, s}$ is definable by a formula $g_{p, s}$. This would require that the logic used for obtaining the formula is expressive enough. This may not always be the case. Even if the logic is expressive enough, computing a formula that exactly defines $T_{p, s}$ may be expensive. On the other hand, it may be easier to compute a formula that defines a super set of $T_{p, s}$. Let $f_{p, s}$ be a formula that defines any superset of $T_{p, s}$. Let $\sim_{0}^{\prime \prime}$ be the relation obtained by replacing the formula $g_{p, s}$ by the formula $f_{p, s}$ in the definition of $\sim_{0}^{\prime}$. Since $g_{p, s} \supset f_{p, s}$ is a valid formula, it is easy to see that $\sim_{0}^{\prime \prime} \subseteq \sim_{0}^{\prime}$ and hence $\sim_{0}^{\prime \prime}$ is a sub-bi-simulation relation. As indicated in sub-section 2.2. we can use $\sim_{0}^{\prime \prime}$ to construct a reduction of $\operatorname{Sym}{ }_{-} \operatorname{Reach}(G, u)$ and perform model checking on this reduction.

\section{Transition Diagrams over Integer Domains}

The use of symbolic graphs can be avoided for certain cases. Consider transition diagrams where the variables are integer variables and the functions updating 
them either increment or decrement them by some constants. Let $f_{c}(x)$ be the function $x+c$ where $c$ is a constant. Obviously for any two constants $c$ and $d$, the functions $f_{c}$ and $f_{d}$ are commutative.

Without constructing the symbolic state graph, we can directly define a bisimulation on the standard reachability graph $\operatorname{Reach}(G, u)$. Recall that each state of $\operatorname{Reach}(G, u)$ is of the form $(q, h)$ where $q \in Q$ and $h$ assigns values to variables in $X$. Let $s=(q, h)$ be a state in $\operatorname{Reach}(G, u)$. For a predicate template $(p, f)$, define $(p, f)[s]$ to be the predicate obtained by replacing each occurrence of a variable $x$ in $p$ by $x+h(f(x))$ if $f(x)$ is a variable. We don't replace $x$ if $f(x)=*$. Let $\approx_{0}$ be the set of pairs of states $(s, t)$, where $s=(q, h)$ and $t=\left(q, h^{\prime}\right)$ for some $q \in Q$, such that for each $(p, f) \in$ ptemplates $(q)$, $(p, f)[s] \equiv(p, f)[t]$ is valid. This construction can be employed for a general case where "+" is any commutative and associative binary operator over the domain of the variables.

Theorem 8. $\approx_{0}$ is a bi-simulation on $\operatorname{Reach}(G, u)$.

Now we show, how to efficiently check if $s \approx_{0} t$ for a TD $G$ where each predicate $p \in \operatorname{guards}(G) \cup A P$ is linear, i.e. is of the form $\Sigma_{1 \leq i \leq n} a_{i} x_{i}>d^{\prime}$ where $x_{i} \in X$ and $a_{1}, \ldots, a_{n}$ are integer constants. Let $s=(q, h)$ and $t=\left(q, h^{\prime}\right)$ be any two states. Let $I=\left\{i: 1 \leq i \leq n\right.$ and $\left.f\left(x_{i}\right) \neq *\right\}$. It is not difficult to see that $(p, f)[s] \equiv(p, f)[t]$ is valid if $\Sigma_{i \in I} a_{i} h\left(f\left(x_{i}\right)\right)=\Sigma_{i \in I} a_{i} h^{\prime}\left(f\left(x_{i}\right)\right)$. This condition can be checked efficiently. It is easy to see that checking equivalence can also be done efficiently when $p$ is of the form $\left(\Sigma_{1<i<n} a_{i} x_{i}\right) \bmod c=c^{\prime}$ where $c, c^{\prime}$ are constants. Thus, if for every predicate $p$ of the form $\Sigma_{1 \leq i \leq n} a_{i} x_{i}>d^{\prime}$ in $\operatorname{guards}(G) \cup A P$, the set $\left\{\Sigma_{i \in I} a_{i} h\left(f\left(x_{i}\right)\right): s=(q, h)\right.$ is a state in $\operatorname{Reach}(G, u)$ and $(p, f) \in$ templates $(q)$ and $I=\{i: f(i) \neq *\}\}$ is finite then $\approx_{0}$ has a finite quotient.

A subclass of integer domain TDs are those in which the variables range over natural numbers; this occurs if all the constants in the transitions are positive and the initial state $u$ assigns only positive values to the variables. Furthermore, we require that each predicate $p$ is a linear predicate and the coefficients of variables in the predicate are all positive. Let $s=(q, h)$ and $t=\left(q, h^{\prime}\right)$ be two states. Now consider any template $(p, f)$ in ptemplates $(s . l c)$ where $p$ is given by $\Sigma_{1 \leq i \leq n} a_{i} x_{i}>d^{\prime}$. Let $I=\left\{i: f\left(x_{i}\right) \neq *\right\}$. It is not difficult to see that $(p, f)[s] \equiv(p, f)[t]$ is valid if either the two values $\Sigma_{i \in I} a_{i} h\left(f\left(x_{i}\right)\right)$ and $\Sigma_{i \in I} a_{i} h^{\prime}\left(\left(f\left(x_{i}\right)\right)\right.$ are equal, or both of them are greater than $d^{\prime}$.

\subsection{Static Analysis for Integer TDs}

\section{Using Linear Equations for Static Analysis}

For any state $s$, any $\left(p\right.$, depend $\left.s_{\pi}\right) \in$ ptemplates $(s . l c)$, we define $T_{p, s}$ to be the set of all $h$ such that, for some $q \in Q_{p}$, the state $(q, h)$ is reachable from the state $(s . l c, \mathbf{0})$ in the Reach $\left(G^{\prime}, u\right)$. Here $Q_{p}$ is the set of nodes as given in the beginning of the previous section and $\mathbf{0}$ is the valuation that gives zero value to all the variables. Note that in the above definition we are considering execution from the state $(s . l c, \mathbf{0})$ not from the state $(s . l c, s . v a l)$ as in the previous section. 
Therefore, $T_{p, s}$ does not depend on the values of the variables in $s$. This set only depends on $p$, i.e., $Q_{p}$ and on s.lc. As given in the previous subsection, using formulas that define the sets of $T_{p, s}$, we can define a bi-simulation $\approx_{0}^{\prime}$ that is bigger than $\approx_{0}$. Such formulas can be obtained by static analysis.

For each $i>0$, we define the bi-simulations $\approx_{i}$ and $\approx_{i}^{\prime}$ just like $\sim_{i}$ and $\sim_{i}^{\prime}$ excepting that these are defined on the structure $\operatorname{Reach}(G, u)$ (they are defined on the same lines as $\approx_{0}$ and $\approx_{0}^{\prime}$ ).

Now we consider a subclass of integer domain TDs in which every assignment of a variable either increments or decrements it by a constant, i.e., a variable is not assigned another variable or a constant. We call such a TD as a restricted TD. Now we give a general procedure for obtaining formulas that define supersets of the sets $T_{p, s}$ as defined above. Observe that these formulas only depend on $p$ and s.lc. Actually, for each $q \in Q$ and for each $r \in Q$, we give a formula $f_{q, r}$ that defines a superset of all valuations $h$ such that the state $(r, h)$ is reachable from $(q, \mathbf{0})$ in $\operatorname{Reach}(G, u)$.

General Procedure for $f_{q, r}$ : Now we give a general procedure to compute $f_{q, r}$ for a restricted TD $G$ where $q, r$ are nodes in $G$. let $\pi$ be a path from $q$ to $r$, for every transition $e$, let $y_{e}$ to denote the number of times $e$ is traversed in the path $\pi$. For every node $u$, let $d^{+}(u)$ denote the set of edges starting at $u$, $d^{-}(u)$ denote the set of edges ending in $u$. Then for every node $u \neq q, r$, we will have $\Sigma_{e \in d^{+}(u)} y_{e}=\Sigma_{e \in d^{-}(u)} y_{e}$. Also we will have $\Sigma_{e \in d^{+}(q)} y_{e}=\Sigma_{e \in d^{-}(q)} y_{e}+1$ and $\Sigma_{e \in d^{+}(r)} y_{e}=\Sigma_{e \in d^{-}(r)} y_{e}-1$. Note that above equations are satisfied by any path from $q$ to $r$. Let $w\left(x_{i}, e\right)$ denote the value by which $x_{i}$ is increased in the transition $e$. In the case $x_{i}$ is not updated in $e$, let $w\left(x_{i}, e\right)=0$. Now Let $x_{i}=\Sigma y_{e} w\left(x_{i}, e\right)$. Let $g_{q, r}$ be the conjunction of all above equations. For any $p \in(A P \cup \operatorname{guards}(G))$ and state $s$, let $e_{p, s}$ be the disjunction of all $g_{p . l c, r}$ such that $r \in Q_{p}$. It should be easy to see that $e_{p, s}$ defines a superset of $T_{p, s}$. As in section 3 . we can use the formulas $e_{p, s}$ to define a sub-bi-simulation relation $\approx_{0}^{\prime \prime}$ similar to $\sim_{0}^{\prime \prime}$, and use it to construct a reduction of $\operatorname{Reach}(G, u)$.

The above procedure, given for restricted TDs, can be extended to any simple TD with minor modifications.

\section{Using Shortest Path Algorithms}

Now, we give a more efficient method for using static analysis in any restricted TD $G$ in which every guard of a transition is a conjunction of conditions of the form $x \# c$ where \# is a relation operator and $c$ is a constant. A cycle in $G$ is a simple path starting and ending in the same node. We say that a cycle is a positive cycle or a negative cycle for a variable $x$ if the sum of all the changes of the value of $x$ along this cycle is positive $(>0)$ or negative $(<0)$ respectively. For any path $\pi$ in $G$ and variable $x$, let weight $(\pi, x)$ denote the sum of the changes in value of $x$ along $\pi$. For any pair of nodes $q, r$ and variable $x$, let $\operatorname{low}(q, r, x), \operatorname{high}(q, r, x)$ be the minimum or maximum of weight $(\pi, x)$ of all paths $\pi$ from $q$ to $r$ respectively. It is easy to see that if there is a path from $q$ to $r$ passing through a negative cycle for $x$, then $\operatorname{low}(q, r, x)$ is $-\infty$; otherwise $\operatorname{low}(q, r, x)$ is a finite number. Similar relation holds for $\operatorname{high}(q, r, x)$ and posi- 
tive cycles. Note that the value $\operatorname{low}(q, r, x)$ or $\operatorname{high}(q, r, x)$ can be computed in polynomial time using shortest path algorithm over graphs 2]. For nodes $q, r$, $f_{q, r}=\wedge_{x \in X} \operatorname{low}(q, r, x) \leq x \leq h i g h(q, r, x)$. As in the earlier paragraph, for any $p \in(A P \cup \operatorname{guards}(G))$ and state $s$, let $e_{p, s}$ be the disjunction of all $f_{s . l c, r}$ such that $r \in Q_{p}$ and $\approx_{0}^{\prime \prime}$ be the corresponding sub-bi-simulation relation. It can be shown that if for every node $q$ and for every variable $x$, all the paths from the initial node to $q$ pass through only positive cycles for $x$ or all such paths pass through only negative cycles, the reduction of $\operatorname{Reach}(G, u)$ with respect to $\approx_{0}^{\prime \prime}$ is finite.

\section{Relaxing the Commutativity Requirement}

So far in this paper, we required that the set of all functions applied to variables of the same type are mutually commutative. This requirement can be relaxed as follows. Recall that for any path $\pi$ in the $G$, depends $s_{\pi}$ is a function that specifies dependencies of values of the variables at the end $\pi$ to their values at the beginning of $\pi$. Now we introduce a binary relation depends_on on variablenode pairs, i.e., pairs of the form $(x, r)$ where $x \in X$ and $r$ is a node in $G$. The pair $(y, r)$ depends_on the pair $(x, q)$ if there exists a path $\pi$ in $G$ from node $q$ to $r$ such that depend $s_{\pi}(y)=x$. It is easy to see that for the TD given in Figure 4, $\left(x, q_{2}\right)$ depends_on $\left(x, q_{1}\right)$ while $\left(x, q_{3}\right)$ does not depends_on $\left(x, q_{2}\right)$ because the only transition from $q_{2}$ to $q_{3}$ assigns a constant to $x$. On the other hand, if $t_{2}$ did not have this assignment for $x$ then $\left(x, q_{3}\right)$ depends_on $\left(x, q_{2}\right)$, and in addition if we had $x:=y$ as the assignment in $t_{1}$ then $\left(x, q_{3}\right)$ depends_on $\left(y, q_{1}\right)$.

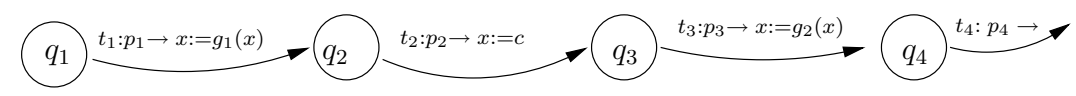

Fig. 4.

Using the relation depends_on, we specify the less restrictive commutative requirement. Let $\Pi$ be the set of all functions that are applied to variables in the transitions of $G$. Let $\left\{\Pi_{1}, \ldots, \Pi_{m}\right\}$ be the finest partition of $\Pi$ which satisfies the following condition: two functions $\psi_{1}$ and $\psi_{2}$ are in the same set $\Pi_{i}$ if there exist pairs $(y, r)$ and $(x, q)$ such that $(y, r)$ depends_on $(x, q)$ and there exist transitions $\left(q^{\prime}, C_{1}, \Lambda_{1}, q\right),\left(r, C_{2}, \Lambda_{2}, r^{\prime}\right)$ such that the assignment $x:=\psi_{1}(x)$ is in $\Lambda_{1}$ and the assignment $y:=\psi_{2}(y)$ is in $\Lambda_{2}$. It is easy to see that the partition can be computed efficiently from $G$ using standard graph algorithms. Consider the example of Figure 4, the functions $g_{1}, g_{2}$ are in the different sets of the partition and hence they need not be commutative. On the other hand, if $t_{2}$ did not have any assignment for $x$ then both $g_{1}, g_{2}$ would be in the same set of the partition and they would need to be commutative. Now, the commutativity requirement is relaxed by requiring that any two functions $f, g \in \Pi$ be commutativity only if they belong to the same set in the partition, i.e., if $f, g \in \Pi_{i}$ for some $i$, $1 \leq i \leq m$. 


\section{Discussion and Related Work}

As indicated in the introduction, this work is an extension of our earlier work [7]. We have essentially extended that work by combining it with static analysis. The static analysis techniques employed here involve the variable value range analysis. Such techniques have been employed earlier in static analysis of programs [6]. Our innovative idea is to combine them with bi-simulation reductions and to employ them in model checking.

The paper [7] discusses the relationship of the original work presented there with the approach given in [5] and with other works based on bi-simulation reduction given in [3]. It is to be noted that none of these works employ static analysis.

\section{References}

1. E. M. Clarke, E. A. Emerson, and A. P. Sistla. Automatic verification of finite state concurrent system using temporal logic specifications: a practical approach. In POPL '83: Proceedings of the 10th ACM SIGACT-SIGPLAN symposium on Principles of programming languages, pages 117-126, New York, NY, USA, 1983. ACM Press.

2. T. H. Cormen, C. E. Leiserson, and R. L. Rivest. Introduction to Algorithms. MIT Press/McGraw-Hill, Cambridge, Massachusetts, 1990.

3. D. Lee and M. Yannakakis. Online minimization of transition systems (extended abstract). In Proceedings of the twenty-fourth annual ACM symposium on Theory of computing, pages 264-274. ACM Press, 1992.

4. Z. Manna and A. Pnueli. The Temporal Logic of Reactive and Concurrent Systems: Specification. Springer-Verlag, Berlin, Jan. 1992.

5. K. S. Namjoshi and R. P. Kurshan. Syntactic program transformations for automatic abstraction. In CAV2000, pages 435-449, 2000.

6. F. Nielson, H. R. Nielson, and C. L. Hankin. Principles of Program Analysis. Springer, 1999.

7. A. P. Sistla, M. Zhou, and X. Wang. Model checking of systems employing commutative functions. In R. Cousot, editor, 6th International Conference on Verification, Model Checking and Abstract Interpretation, Lecture Notes in Computer Science. Springer, 2005. 